\title{
Modelling of Coupling between Mechanics and Water Diffusion in Bonded Assemblies
}

\author{
C. Valançon', A. Roy' and J.C. Grandidier' \\ 1 LMPMUMR 6617 ENSMA, Téléport 2, 1, avenue Clément Ader, B.P. 40109, F-86961 Futuroscope - France \\ 2 CRITT Matériaux, B.P. 1 15, avenue Marcel Dassault, 17303 Rochefort Cedex - France \\ e-mail: valancon@lmpm.ensma.fr - grandidier@Impm.ensma.fr - a.roy@crittmateriaux.com
}

\begin{abstract}
Résumé - Modélisation du couplage entre la mécanique et la diffusion de l'eau dans les assemblages collés - Les adhésifs structuraux sont des polymères amorphes exhibant un comportement viscoélastique. Ces colles étant utilisées dans des conditions très proches de leur température de transition vitreuse, leur comportement est alors piloté par l'évolution du volume libre. Les équations régissant le problème thermo-diffuso-mécanique dérivent des principes fondamentaux de la mécanique des milieux continus et de la thermodynamique des processus irréversibles. La viscoélasticité et les variations de volume libre seront traitées à l'aide du modèle de viscoélasticité non linéaire de Knauss et Emri [Polym. Eng. Sci., 27, 86-100]. Les hypothèses de matériau isotrope et de petites déformations seront faites.
\end{abstract}

\begin{abstract}
Modelling of Coupling between Mechanics and Water Diffusion in Bonded Assemblies Structural adhesives are essentially amorphous polymers exhibiting viscoelastic behaviour. These glues are used near their glassy transition temperature. In this temperature range, their behaviour is driven by free volume evolutions. Equations governing thermo-diffuso-mechanical problem derive from the fundamental principles of continuum mechanics and thermodynamics of irreversible processes. Viscoelasticity and free volume variations are treated by the non linear viscoelastic modelling of Knauss and Emri [Polym. Eng. Sci., 27, 86-100] in an isotropic and small strain framework.
\end{abstract}




\section{INTRODUCTION}

In several sectors of industry, structural bonding is of great interest. It is more and more used to assemble pieces participating to structural resistance. This process is, for example, widely exploited by nautical sector. Indeed, the bulkheads and the hull of a boat are bonded and the assembly is submitted to many solicitations which can be harmful. During its life, a joint must support cycles of mechanical and environmental loadings. Sudden temperature variations but also humidity variations accelerate adhesive aging and reduce its resistance.

In a previous study [1], an assembly bonded joint was held in water at a temperature near the $T g$ of the adhesive and a mechanical load was applied. First, water diffuses through free surfaces and interfaces. The adhesive gradually absorbs water and swells until failure occurs. It has been suggested that stress gradient accelerates water diffusion but also that diffusion is driven by this gradient. Thus it seems paramount to consider the coupling between mechanics and diffusion with the aim of predicting assembly bonded joint rupture.

Water presence has already been modelled considering a diffusion coefficient depending on temperature and water concentration [2] or on temperature and free volume [3]. These studies are able to give mechanical behaviour of resins. However, they are unable to describe the coupling phenomenon shown earlier. To describe completely the problem of water diffusion in an adhesive, one must elaborate a model considering all phenomena: diffusion, thermics and mechanics. In this paper, we present a first model considering all couplings. These couplings will be separated here in to two families:

- Couplings refered to as direct, such as the influence of stress gradient on the diffusion path, thermal dilatation and expansion generated by diffusion on volume strain.

- Couplings refered to as indirect, such as the influence of absorbed water on creep response taken into account through functions of material parameters such as state variables and internal variables.

This modelling is essentially phenomenological, but it is based on some physical considerations which are essential. Most adhesives used in industry are thermosetting amorphous polymers. Generally, they are used in the vicinity of their glassy transition temperature. Their behaviour is then driven by the free volume [4]. In the literature, it is proposed that this free volume is sensitive to temperature, mechanical solicitations and water absorbed by the material [5]. On these physical assumptions, we introduce indirect couplings in the formulation. Free volume evolution influences viscous behaviour of polymer in modifying relaxation times. Water and polymer are considered as a perfect and homogeneous mixture [6]. To describe direct couplings, the model is built in the framework of thermodynamics of irreversible processes [7].

This article presents balance equations and a "diffusothermo-mechanical" constitutive law, describing principally the behaviour of bonded joints of thermosetting resins on a moist environment. First numerical results on the structural relaxation will be compared to literature data.

\section{BALANCE EQUATIONS}

\subsection{Mass Balance}

Consider a polymer absorbing water. These two species form a perfect, homogeneous and continuous mixture [6] where chemical reactions are neglected. The following relationship is then respected:

$$
Y^{w}+Y^{p}=1
$$

where $Y^{w}$ and $Y^{p}$ are respectively specific fraction of water and polymer. Equations will in the following be expressed only as a function of water fraction. Polymer behaviour can then be deduced through Equation (1). Studied materials are rather sensitive to hydrolysis phenomena. Nevertheless, to simplify the problem, we assume there is no chemical reaction between water and polymer.

Consider a representative volume of this mixture. The mass contained in this volume varies only by matter transfer through its walls. This phenomenon is described by the equation of mass balance:

$$
\rho \dot{Y}^{w}=-\operatorname{div} \overrightarrow{J^{m w}}
$$

where $\rho$ is mixture density and $\overrightarrow{J^{m \omega}}$ is vector of mass flux relating to water. In the following, we use the notation '.' for all time derivatives.

\subsection{Momentum Balance}

Balance equations derived from fundamental principle of mechanics are written in their local form:

$$
\overrightarrow{d i v} \underline{\underline{\sigma}}+\rho \vec{f}=\rho \vec{\gamma}
$$

where $\underline{\underline{\sigma}}$ is stress tensor of Cauchy applied to the mixture, $\vec{f}$ represents specific density of forces exerted identically on each volume point. Adhesive ageing is our primary interest here. Therefore, acceleration $\vec{\gamma}$ exerted on each particle of the mixture is neglected.

\subsection{Energy Conservation - First Principle}

Total energy conservation of the volume (first principle of thermodynamics) is expressed classically in its local form by: 


$$
\rho \dot{u}=\underline{\underline{\sigma}}: \underline{\underline{\varepsilon}}-\operatorname{div} \overrightarrow{J^{q}}+r
$$

where $u$ is specific internal energy of the mixture, $\underline{\underline{\varepsilon}}$ linearised strain tensor, $\overrightarrow{J^{q}}$ heat flux vector relating to heat given by conduction and $r$ volume density of heat due to external sources.

\subsection{Second Principle of Thermodynamics}

The main point in this modelling is to introduce and define a set of internal variables. First, it seems important to note that, after a fast quench below $T_{g}$, when temperature equilibrium is reached, material volume evolves slowly towards its stable state [8]. The same phenomenon is found when pressure is considered. An isopiestic volume evolution is found [4]. This is the consequence of free volume relaxation. One can then extend structural relaxation to water diffusion. More precisely, a sudden jump in water concentration below $T g$ takes the material out of equilibrium. When macroscopic equilibrium is reached and maintained, the material continues to evolve towards a stable state. Relaxation of each process is treated through distinct internal variables characterising the evolution of all the different microscopic processes. For homogeneity in notations, mathematical representation of internal variables is chosen with respect to the corresponding state variables.

Most adhesives used for structural bonding are amorphous polymers cross-linking under ambient conditions. At ambient temperature, adhesives can be in a temperature range near their glassy transition temperature. In this range, molecular mobility is making easier by free volume presence. This latter is a direct function of hydrostatic pressure. Consequently, volume and deviatoric effects must be differentiated. These are taken into account considering separately the hydrostatic part $(\operatorname{tr} \underline{\underline{\sigma}})$ and the deviatoric part $(\underline{\underline{s}})$ of the stress tensor. Thus, by mathematical coherence, strain tensor and internal variables tensor are decomposed into their traces ( $\operatorname{tr} \underline{\underline{\varepsilon}}$ and $\mathrm{z}^{\mathrm{tr} \varepsilon}$ ) and their deviators ( $\underline{\underline{e}}$ and $\underline{\underline{z}}^{e}$ ). For temperature and diffusion phenomena, scalar internal variables are introduced, respectively $z^{T}$ and $z^{c}$. This choice of separating internal variables means that, when water diffusion in the material occurs, physical mechanisms taking place are different from those acting due to mechanical loading. This decision can be questioned because in the material, it is molecular mobility which is the main microscopic mechanism. Nevertheless, diffusion phenomenon refers to chemical considerations which have another origin than "crank" mechanisms (see below for a discussion of "crank" mechanisms). Internal variables introduced here allow us to quantify the deviation from a diffuso-thermo mechanical equilibrium state. Out theoretical framework is the thermodynamics of irreversible processes [7] where one considers a medium which is open and in course of evolution. Based on the second principle, intensive variables (internal energy $u$, entropy $s$, stress tensor of Cauchy $\underline{\underline{\sigma}}$, difference of chemical potentials $\Delta \mu=\left(\mu^{\mathrm{w}}-\mu^{\mathrm{p}}\right)$ and thermodynamical affinities associated with each elementary process $\mathrm{A}^{\mathrm{Tj}}, \mathrm{A}^{\mathrm{cj}}, \mathrm{A}^{\mathrm{tr \varepsilon j}}$ and $\stackrel{A^{e j}}{=}$ ) are related to their respective extensive variables (temperature $T$, linearised strain tensor $\underline{\underline{\varepsilon}}$, specific fraction of water $Y^{w}$ and introduced internal variables $z^{T j}, z^{c j}, z^{t r \varepsilon j}$ and $z^{e j}$ ).

$$
\begin{aligned}
& T \dot{s}=\dot{u}-\frac{1}{\rho}(\operatorname{tr} \underline{\underline{\sigma}})(\operatorname{tr} \underline{\underline{\varepsilon}})-\frac{1}{\rho} \underline{\underline{s}}: \underline{\underline{\dot{e}}}-\Delta \mu \dot{Y}^{w} \\
& +\sum_{j=1}^{n} A^{t r \varepsilon j} \dot{z}^{t r \varepsilon j}+\sum_{j=1}^{k} \underline{\underline{A}}^{e j}: \underline{\underline{z}}^{e j}+\sum_{j=1}^{n} A^{T j} \dot{z}^{T j}+\sum_{j=1}^{n} A^{c j} \dot{z}^{c j}
\end{aligned}
$$

One distinguishes two contributions in entropy variation: one giving by exchanges with the exterior $\dot{s}_{\mathrm{e}}$ and another due to internal sources $\dot{s}_{\mathrm{i}}$ such as: $\rho \dot{s}=\rho \dot{s}_{\mathrm{e}}+\rho \dot{s}_{\mathrm{i}}$ with $\dot{s}_{\mathrm{i}} \geq 0$.

The variation of internal entropy defines volume dissipation $\varphi$ via Equation (6):

$$
\begin{aligned}
& \rho T \dot{s}_{i}=\rho \sum_{j=1}^{n} A^{t r \varepsilon j} \dot{z}^{t r e j}+\rho \sum_{j=1}^{k} A^{e j}: \dot{z}^{e j}+\rho \sum_{j=1}^{n} A^{T j} \dot{z}^{T j}+\rho \sum_{j=1}^{n} A^{c j} \dot{z}^{c j} \\
& -\overrightarrow{J^{s}} \cdot \overrightarrow{\operatorname{grad} T}-\overrightarrow{J^{m w}} \cdot \overrightarrow{\operatorname{grad}} \Delta \mu \geq 0
\end{aligned}
$$

where $\overrightarrow{J^{s}}$ is vector of entropy flux which is related to heat

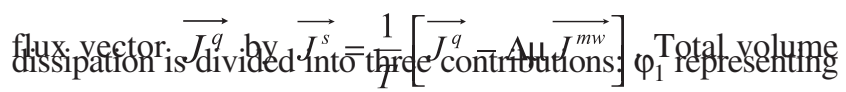
dissipation due to internal processes, $\varphi_{2}$ volume dissipation due to thermics and $\varphi_{3}$ giving by diffusive phenomenon.

$$
\begin{gathered}
\varphi_{1}=\rho \sum_{j=1}^{n} A^{t r \varepsilon j} \dot{z}^{t r \varepsilon j}+\rho \sum_{j=1}^{k} A^{e j}: \underline{\dot{z}}^{e j}+\rho \sum_{j=1}^{n} A^{T j} \dot{z}^{T j}+\rho \sum_{j=1}^{n} A^{c j} \dot{z}^{c j} \\
\varphi_{2}=-\overrightarrow{J^{s}} \cdot \overrightarrow{\operatorname{grad} T} \\
\varphi_{3}=-\overrightarrow{J^{m w}} \cdot \overrightarrow{\operatorname{grad}} \Delta \mu
\end{gathered}
$$

\section{CONSTITUTIVE EQUATIONS}

\subsection{Complementary Laws of Evolution}

For the sake of simplicity, the principle of Curie is applied. Only a partial coupling is then envisaged for establishing complementary laws of evolution (i.e. only coupling between diffusive and thermal phenomenon is considered). Direct coupling relating vectors of specific flux of water and entropy flux to gradients of temperature and difference of chemical potentials are presented in the following classical form:

$$
\overrightarrow{J^{s}}=\underline{\underline{k}}^{T} \cdot[\overrightarrow{\operatorname{grad}} T]+\underline{\underline{c}}_{T \mu} \cdot[\overrightarrow{\operatorname{grad}} \Delta \mu]
$$




$$
\overrightarrow{J^{m w}}=\underline{\underline{k}}_{\mu} \cdot[\overrightarrow{\operatorname{grad}} \Delta \mu]+\underline{\underline{c}}_{T \mu} \cdot[\overrightarrow{\mathrm{grad}} T]
$$

where $\underline{k}^{T}, \underline{k}_{\mu}$ and $\stackrel{c}{=}_{T \mu}$ are coupling coefficients of Onsager.

Concerning internal variables, they translate viscoelastic characteristics of amorphous polymers. As has often been emphasized before, adhesives are used in a temperature range near their glassy transition temperature. In this range, polymers behaviour is driven by free volume. The latter influences relaxation times in the matter. In the non linear viscoelastic model realised by Knauss and Emri [5], evolution laws of relaxation times are proposed and seem to be efficient. Based on these developments we propose a family of evolution laws of internal variables. These laws generalize the model of Knauss and give a thermodynamical justification based on mechanics to extend this concept to a case of diffusion, thermics and mechanics:

$$
\begin{aligned}
& \dot{z}^{\text {trej }}=-\frac{1}{\tau_{\text {volume }}^{\text {trej }} a^{\text {tre }}{ }_{j}\left(\operatorname{tr} \underline{\underline{\varepsilon}}, T, Y^{w}\right)} \times \\
& \left\{\left(z^{t r \varepsilon j}-z_{\infty}^{t r \varepsilon j}\right)+\alpha_{j}^{T}\left(z^{T j}-z_{\infty}^{T j}\right)+\alpha_{j}^{c}\left(z^{c j}-z_{\infty}^{c j}\right)\right\} \\
& \dot{z}^{T j}=-\frac{1}{\tau_{\text {volume }}^{T j} a^{T j}\left(\operatorname{tr} \underline{\underline{\varepsilon}}, T, Y^{w}\right)} \times \\
& \left\{\left(z^{T j}-z_{\infty}^{T j}\right)+\frac{T_{o} k_{j} \alpha_{j}^{T}}{\rho C_{j}^{p}}\left(z^{t r \varepsilon j}-z_{\infty}^{t r \varepsilon j}\right)+\frac{T_{o} k_{j} \alpha_{j}^{c}}{\rho C_{j}^{p}}\left(z^{c j}-z_{\infty}^{c j}\right)\right\} \\
& \dot{z}^{c j}=-\frac{1}{\tau_{\text {volume }}^{c j} a^{c j}\left(\operatorname{tr} \underline{\underline{\varepsilon}}, T, Y^{w}\right)} \times \\
& \left\{\left(z^{c j}-z_{\infty}^{c j}\right)+\frac{d_{j}^{T}}{d_{j}^{c}}\left(z^{T j}-z_{\infty}^{T j}\right)+\frac{k_{j} \alpha_{j}^{c}}{d_{j}^{c}}\left(z^{t r \varepsilon j}-z_{\infty}^{t r \varepsilon j}\right)\right\} \\
& \dot{\underline{\underline{z}}}^{e j}=-\frac{1}{\tau_{\text {shear }}^{e j} a^{e j}\left(\operatorname{tr} \underline{\underline{\varepsilon}}, T, Y^{w}\right)}\left(\underline{\underline{\underline{e}}}^{e j}-\underline{\underline{z}}_{\infty}^{e j}\right)
\end{aligned}
$$

where indices $\infty$ and $j$ refer respectively to equilibrium state and different state in the course of evolution,

$\tau$ is characteristic relaxation time at each internal state,

$a$ is time shift function,

$\alpha_{j}^{T}$ coefficient of thermal dilation,

$\alpha_{j}^{c_{j}}$ coefficient of dilation produced by solvent diffusion,

$T_{o}$ is initial temperature,

$k_{j}$ is bulk modulus of the $j$-th internal state,

$C^{p}{ }_{j}$ specific heat of the $j$-th internal state,

$d_{j}^{T}$ diffusion coefficient relating to thermics,

$d_{j}^{c}$ diffusion coefficient relating only to water presence in the polymer.
From internal point of view, one knows that molecular segments move essentially by jumps called "cranks". For a jump to take place, the intermolecular space, named also free volume, must be sufficient. Jump activation is generally the result of thermal vibrations. Imagine now that internal variable $z^{T}$ represents the thermal vibration of a molecular jump, Equation (13) tells us that this vibration depends on a thermal part necessary to reach an equilibrium state but is also disrupted by relaxation phenomenon provided by mechanical and diffusive loadings. Internal variables relating to mechanics must be associated to free volume fraction or excess of entropy. The evolution of this latter is indeed the consequence of internal relaxations. When macroscopic equilibrium is reached, the material continues to evolve. Equation (12) describes this phenomenon.

\subsection{Relaxed State}

The evolutions of dissipative internal processes can be understood by the differential equations presented above. Internal variables translating the deviation from equilibrium tend to this state when they are not excited. Equilibrium definition is not easy because it is hypothetical. Relaxation times of considered materials can be such that the relaxed state is reached in a time scale above reasonable experimental time scale. In a first approach, we have chosen tangential linear laws for each family of internal variables.

$$
\begin{array}{ll}
d z_{\infty}^{t r \varepsilon j}=\beta^{t r \varepsilon j} d(\operatorname{tr} \underline{\underline{\varepsilon}}) & d z_{\infty}^{T j}=-\beta^{T j} d T \\
d z_{\infty}^{c j}=-\beta^{c j} d Y^{w} & d \underline{\underline{z}}_{\infty}^{e j}=\beta^{e j} d \underline{\underline{e}}
\end{array}
$$

where coefficients $\beta$ are rigidities of relaxed state.

\subsection{State Laws}

The thermodynamical potential chosen to define state laws is specific free energy. This is taken as a simple quadratic and isotropic function of macroscopic data and internal variables. One presents only state laws, which are the derivatives of the thermodynamical potential, introducing the rigidities of relaxed states by the use of the equilibrium definition of internal variables (16). Coupling between each phenomenon is taken into account in a relatively complete manner. However, indirect coupling of constitutive laws is done through free volume. Its evolution is essentially influenced by volume changes. In this framework, we have chosen not to describe direct coupling between shear behaviour and others phenomena. On the other hand, relaxation times in shear are affected by these latter. Only constitutive laws concerning stresses and chemical potentials are presented. 


$$
\begin{aligned}
& \operatorname{tr} \underline{\underline{\sigma}}=k_{\infty} \operatorname{tr} \underline{\underline{\varepsilon}}-k_{\infty} \alpha_{\infty}^{T}\left(T-T_{o}\right)-k_{\infty} \alpha_{\infty}^{c}\left(Y^{w}-Y_{o}^{w}\right) \\
& -\sum_{j=1}^{n} \beta^{t r \varepsilon j} k_{j}\left(z^{t r \varepsilon j}-z_{\infty}^{t r \varepsilon j}\right)+\sum_{j=1}^{n} \beta^{t r \varepsilon j} k_{j} \alpha_{j}^{T}\left(z^{T j}-z_{\infty}^{T j}\right) \\
& +\sum_{j=1}^{n} \beta^{t r \varepsilon j} k_{j} \alpha_{j}^{c}\left(z^{c j}-z_{\infty}^{c j}\right) \\
& \underline{\underline{s}}=2 * \underline{\underline{\underline{G_{\infty}}}}: \underline{\underline{e}}-2 \sum_{j=1}^{k} \beta^{e j} \underline{\underline{\underline{\underline{G_{j}}}}}:\left(\underline{\underline{z^{e j}}}-\underline{\underline{z^{e j}}}\right) \\
& \rho \Delta \mu=d_{\infty}^{c}\left(Y^{w}-Y_{o}^{w}\right)-k_{\infty} \alpha_{\infty}^{c}(t r \underline{\underline{\varepsilon}})-d_{\infty}^{T}\left(T-T_{o}\right) \\
& -\sum_{j=1}^{n} \beta^{c j} d^{c j}\left(z^{c j}-z_{\infty}^{c j}\right)-\sum_{j=1}^{n} \beta^{c j} k_{j} \alpha_{j}^{c}\left(z^{t r \varepsilon j}-z_{\infty}^{t r \varepsilon j}\right) \\
& -\sum_{j=1}^{n} \beta^{c j} d^{T j}\left(z^{T j}-z_{\infty}^{T j}\right)
\end{aligned}
$$


librium state and $\overline{\overline{\bar{j} \text {-th }}}$ state in course of evolution.

One notes that, in the expression for stress, usual terms are found such as strain and temperature but now diffusion occurs through specific inclusion of the fraction of water and diffusion processes in the course of evolution. One finds also the fact that the material changes instantaneously in volume but also with retardation effects. In the same way, the difference of chemical potentials which represents the driving force for diffusion depends on the quantity of absorbed water but also on the repercussions of mechanics and thermics on rearrangement of molecular segments.

\subsection{Equation of Diffusion}

Finally, in considering Equations (2), (11) and (19) the vector of specific flux of water is presented in the following form:

$$
\begin{aligned}
& \overrightarrow{J^{m w}}=\underline{\underline{k}}_{\mu}\left[-\frac{1}{\rho}\left(d_{\infty}^{c} 10^{6} s^{e} \overrightarrow{g r a d} c-\sum_{j=1}^{n} \beta^{c j} d_{j}^{c} \overrightarrow{\operatorname{grad}}\left(z^{c j}-z_{\infty}^{c j}\right)\right.\right. \\
& -k_{\infty} \alpha_{\infty}^{c} \overrightarrow{\operatorname{grad}}(\operatorname{tr} \underline{\underline{\varepsilon}})-\sum_{j=1}^{n} \beta^{c j} k_{j} \alpha_{j}^{c} \overrightarrow{\operatorname{grad}}\left(z^{t r \varepsilon j}-z_{\infty}^{t r \varepsilon j}\right) \\
& \left.\left.-\sum_{j=1}^{n} \beta^{c j} d_{j}^{T} \overrightarrow{\operatorname{grad}}\left(z^{T j}-z_{\infty}^{T j}\right)\right)\right]+\left(\underline{\underline{c}}_{T \mu}-\frac{\underline{\underline{k}}_{\mu} d_{\infty}^{T}}{\rho}\right)[-\overrightarrow{\operatorname{grad}} T]
\end{aligned}
$$

where $s^{e}$ is water solubility and $c$ is water concentration.

The first term of specific flux matches the classical law of Fick. The second term is not classical but corresponds to internal phenomenon of relaxation relating to water diffusion. The third and fourth terms represent the influence of material volume variation on flux of mass. More precisely, these terms represent the effect of spatial reparti- tion of hydrostatic stress (bulk modulus in product with volume strain) on the diffusion and on the direction of diffusion. This permits to explain through couplings the observations of Roy et al. [1], which suggest that stress gradient accelerates water diffusion but also that diffusion is driven by this gradient. The fourth term represents the volume relaxation effect producing by mechanics on specific flux. In the same way, the last terms of the specific flux translate the effect of thermal gradient on the diffusion path. It is clearly established that temperature influences diffusion kinetics. Nevertheless, here, it is the temperature gradient which directly influences diffusion path. This point needs thus to be verified experimentally.

Following observations made during earlier works [1], one can hope that this modelling contains all ingredients to represent quantitatively experimental phenomenon.

\section{NUMERICAL RESULTS}

To first improve this modelling, a software has been developed in Fortran and only considering the constitutive laws for the stress $(17,18)$. Therefore, numerical test can be only about volume variations and the phenomenon of structural relaxation is chosen. More precisely, the relaxation after relative humidity jumps is studied and compared to the experimental results of Zheng and McKenna [9]. Specimens used in their experiments are epoxy membranes which are put in a controlled humidity room at a constant temperature. Jumps are done from a relative humidity $(90 \%)$ above $\mathrm{RHg}(60 \%)$ to relative humidity below (reported in Fig. 1) at a rate of $1.5 \% / \mathrm{min}$. In Figure 1 is represented the evolution of the departure $\delta$ from the equilibrium during the soaking. This quantity is defined as follows:

$$
\delta=\frac{V-V_{\infty}}{V_{\infty}}
$$

and in the presented model as a function of $\operatorname{tr} \underline{\underline{\varepsilon}}$ :

with

$$
\begin{gathered}
\delta=\operatorname{tr} \underline{\underline{\varepsilon}}(R H, t)-\operatorname{tr} \underline{\underline{\varepsilon}}(R H, \infty) \\
\operatorname{tr} \underline{\underline{\varepsilon}}(R H, \infty)= \pm \alpha_{\infty}^{c} \Delta R H
\end{gathered}
$$

where $V$ denotes the specific volume, $V_{\infty}$ the equilibrium volume and $R H$ the relative humidity rate.

A comparison between numerical results and literature data [9] is illustrated in Figure 1. First, the model gives a good approximation of the phenomenon of the structural relaxation provoked by a $R H$-jump. The departure at the beginning of the soaking is not very well estimate and the evolution of the volume for the smallest jump is overestimated. Nevertheless, these results show the ability of the presented model to represent physical phenomenon such as structural relaxation. Other simulations must be realised to validate the model entirely. 


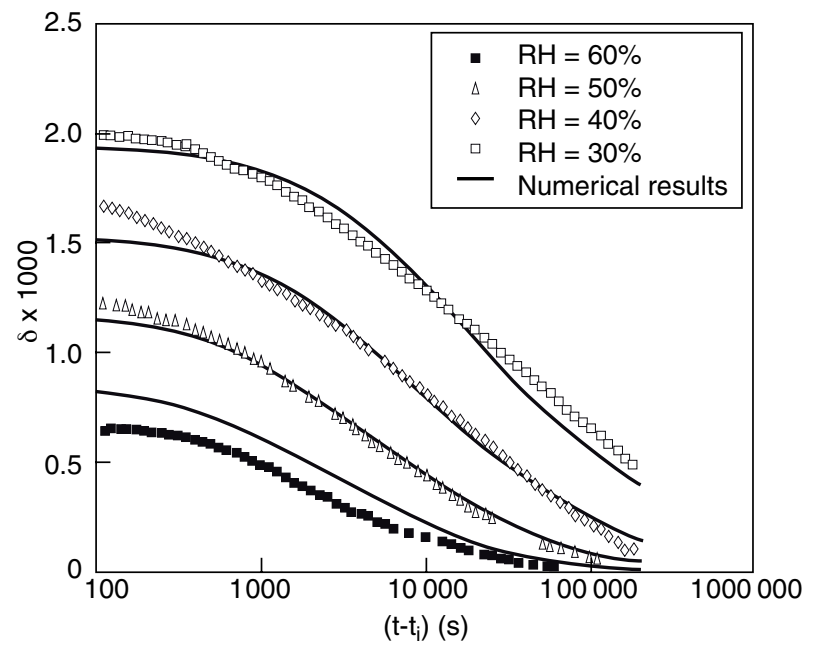

Figure 1

Comparison between numerical results and experiments of Zheng and McKenna [9].

\section{CONCLUSION}

In this article, the main equations of a model considering a thermo-diffuso-mechanical coupling have been developed. This latter has essentially been built for describing the behaviour of thermosetting amorphous polymers, and more precisely structural adhesive. A first comparison between numerical results and experimental results of literature has been presented and gives a good fit, but other investiga- tions must be done to validate this model. One can imagine that the application range can be expanded to all amorphous polymers and to several species in diffusion.

\section{REFERENCES}

1 Roy, A., Gontcharova-Bénard, E., Gacougnolle, J.-L. and Davies, P. (2000) Hygrothermal effects on failure mechanisms of composite/steel bonded joints, in Time dependent and nonlinear effects in polymers and composites, ASTM STP 1357, Schapery, R.A. and Sun, C.T. (Eds.), American society for testing and materials, West Conshohocken, PA.

2 Weitsman, Y. (1990) A Continuum diffusion model for viscoelastic materials., J. Phys. Chem., 94, 961-968.

3 Roy, S. and Reddy, J.N. (1988) A finite element analysis of adhesively bonded composite joints with moisture diffusion and delayed failure, Comput. Struct., 29, 1011-1031.

4 Kovacs, A.J. (1966) Applicability of the free volume concept on relaxation phenomena in the glass transition range, Rheol. Acta, 5, 262-269.

5 Knauss, W.G. and Emri, I. (1987) Volume change and the nonlinearity thermo-viscoelastic constitution of polymers, Polym. Eng. Sci., 27, 86-100.

6 Müller, I. (1975) Thermodynamics of mixtures of fluids, J. Mécanique, 14, 268-303.

7 Münster, A. (1966) Thermodynamique des processus irréversibles, Bibliothèque des Sciences et Techniques Nucléaires.

8 Kovacs, A.J. (1958) La contraction isotherme du volume des polymères amorphes, J. Polym. Sci., 30, 131-147.

9 Zheng, Y. and McKenna, G.B. (2003), Structural recovery in a model epoxy: Comparison of responses after temperature and relative humidity jumps, Macromolecules, 36, 1164-1169.

Final Manuscript received in October 2006 